\section{Retinal pigment epithelial tears after single administration of intravitreal bevacizumab for neovascular age-related macular degeneration}

Center for Ophthalmology, University of Tuebingen,

Tuebingen, BW, Germany

Correspondence:

F Gelisken, Center for

Ophthalmology, University

of Tuebingen, Schleichstr.

12, Tuebingen, BW 72076,

Germany.

Tel: + 4970712984761 ;

Fax: + 497071294763.

E-mail: faik.gelisken@

med.uni-tuebingen.de

Received: 11 July 2007 Accepted in revised form: 19 December 2007

Published online: 1 February 2008

Financial interest:

None

This article was presented in part at the combined Meeting of Club Gonin and The Retina Society, October 2006, Cape Town.
Purpose To analyse retinal pigment epithelial (RPE) tears following single administration of intravitreal bevacizumab for neovascular agerelated macular degeneration (AMD) during early follow-up.

Methods Interventional, retrospective, noncomparative case series included 397 patients (409 eyes) of the 746 consecutive patients that met the eligibility criteria. Standardized visual acuity testing, fluorescein angiography, and optical coherence tomography were performed. Data collected included status of the fellow eye, previous treatment, subtypes of choroidal neovascularization (CNV), size and composition of the lesion. Multiple linear regression modelling was used to explore the effect of baseline parameters on the RPE tears. Primary end point was occurrence of RPE tears within 6 weeks after therapy.

Results Fifteen of the 409 eyes (3.6\%) developed RPE tear $(95 \%$ confidence interval: 2.2-6.0, odds ratio: 26.3). The statistical modelling showed significant association between RPE tear and occult without classic $\mathrm{CNV} /$ predominantly haemorrhage $v$ s predominantly/minimal classic $\mathrm{CNV}$ $(P=0.019)$, as well as medium or large ( $>4$ disc area) $v s$ small size of the total lesion $(P=0.038)$. Previous treatment and status of the fellow eye did not statistically influence the risk of RPE tears.

Conclusions An RPE tear can develop in up to $3.6 \%$ of eyes with neovascular AMD following single administration of intravitreal

\section{Abstract}

F Gelisken, F Ziemssen, M Voelker,

KU Bartz-Schmidt and W Inhoffen bevacizumab in a short-term follow-up. Medium and large lesion size and occult without classic and predominantly haemorrhagic subtype of $\mathrm{CNV}$ were important predictive factors. Preoperative assessment of the lesion characteristics may help in identifying the risk of individual patients with neovascular AMD before intravitreal bevacizumab treatment.

Eye (2009) 23, 694-702; doi:10.1038/sj.eye.6703098; published online 1 February 2008

Keywords: age-related macular degeneration; bevacizumab; choroidal neovascularization; complication; retinal pigment epithelial tear; vascular endothelial growth factor

\section{Introduction}

Neovascular age-related macular degeneration (AMD) is the leading cause of central vision loss in industrialized countries. ${ }^{1}$ The major role of the vascular endothelial growth factor (VEGF) in the pathogenesis of the choroidal neovascularization $(\mathrm{CNV})$ had been highlighted. ${ }^{2-4}$ A beneficial effect of intravitreal therapy with anti-VEGF for neovascular AMD has been shown by recent reports of randomized controlled clinical studies..$^{5-7}$

Bevacizumab (Avastin, Genentech Inc., San Francisco, CA, USA) is a recombinant monoclonal antibody, inhibiting human VEGF. Even though originally approved by the Food and Drug Administration for the therapy of metastatic colorectal cancer, intravitreal 
bevacizumab administration has gained popularity in the therapy of neovascular AMD. ${ }^{8-11}$ The long-term safety and efficacy of intravitreal bevacizumab application in neovascular AMD has not been assessed in randomized controlled clinical trials; there is even a need for a clinician and for a selection of suitable patients.

Experimental and clinical research did not demonstrate intraocular toxicity of this drug. ${ }^{12-14}$ In contrast, some cases of retinal pigment epithelial (RPE) tears after bevacizumab therapy have recently been reported. ${ }^{15-26}$ Since intravitreal bevacizumab is a widely used therapy option for neovascular AMD, there is a need for further information on this topic. We analysed the occurrence of the RPE tear and the influence of related factors on this entity in a short-tem follow-up period following a single intravitreal bevacizumab administration for neovascular AMD.

\section{Materials and methods}

After obtaining institutional ethics committee approval, the medical charts of patients were reviewed retrospectively. All patients with neovascular AMD were screened who underwent intravitreal bevacizumab injection between September 2005 and December 2006 at the Center for Ophthalmology of the University of Tuebingen. Written informed consent was given by all patients before treatment.

Inclusion and exclusion criteria are given in Table 1. Eyes were included with clinical and fluorescein angiographic evidence of CNV secondary to AMD.

Table 1 Inclusion and exclusion criteria

Inclusion criteria
Age $>50$ years
AMD
Clinical and fluorescein angiographic evidence of CNV
Available post-treatment follow-up within 6 weeks
( \pm 2 weeks)
Written informed consent for treatment
Eyes with or without previous treatment of CNV
All lesion types of CNV
Exclusion criteria
Missed follow-up
Inadequate photographic documentation
Retinal angiomatous proliferation
Polypoidal choroidal vasculopathy
Retinal pigment epithelial tear
Pure serous PED
Combined therapy
Long standing disciform macular scars

Abbreviations: $\mathrm{AMD}=$ age-related macular degeneration; $\mathrm{CNV}=$ choroidal neovascularization; PED = pigment epithelial detachment.

a Defined as an additional therapy for the CNV within 1 week of the intravitreal bevacizumab injection.
All subtypes of CNV without restriction of size and previous therapy were allowed. Exclusion criteria were eyes with an RPE tear at baseline, missed follow-up, inadequate documentation, retinal angiomatous proliferation (RAP), polypoidal choroidal vasculopathy (PCV), and combined therapy with bevacizumab.

Ophthalmic examination at baseline and at follow-up visit within 6 weeks ( \pm 2 weeks) after the treatment included standardized best-corrected visual acuity (BCVA) testing using Early Treatment of Diabetic Retinopathy charts, fundus photography, and optical coherence tomography (OCT). The diagnosis of the RPE tears was based on the slit-lamp ophthalmoscopy with dilated pupil, fundus photography, and OCT imaging of the macula at 6 weeks control examination. Fluorescein angiography at 6 weeks control was performed if an RPE tear was suspected or change of the therapy was considered. All reported eyes with RPE tear had a fluorescein angiography. Special attention was given to differentiate PCV and RAP. Indocyanine green (ICG) angiography was performed if ophthalmoscopy and fluorescein angiogram were suspicious for PCV or RAP lesions. All cases with PCV and the majority of RAP lesions were confirmed by ICG angiography. Before the injection, a $10 \%$ solution of povidione iodine was applied on the eyelid margins and a 5\% solution of povidione iodine on the conjunctival surface. After topical anaesthesia and covering the eye by a sterile drape, a lid speculum was inserted. Intravitreal injection of $0.5 \mathrm{ml}$ $(1.25 \mathrm{mg})$ bevacizumab with a 27 -gauge needle was administered through a distance of $3.5 \mathrm{~mm}$ from the limbus.

Data collected include age, sex, previous therapy of the $\mathrm{CNV}$, and status of the fellow eye. Fluorescein angiographic subtype of $\mathrm{CNV}$ and lesion characteristics was also determined. Fluorescein angiographic interpretation of the CNV lesion and lesion components was based on the modified definition of Macular Photocoagulation Study Group and guidelines for using photodynamic therapy with verteporfin for neovascular AMD. ${ }^{27,28}$ Additionally, eyes were included if the size of the submacular haemorrhage was larger than the CNV lesion. This subgroup was classified as predominantly haemorrhagic. Lesion components include features that obscure the boundaries of $\mathrm{CNV}$, such as haemorrhage, subretinal fibrin, fibrosis, or serous pigment epithelial detachment (PED). All lesion components and CNV were marked and measured digitally by a masked review process using a software package supported by Zeiss (Visupac Software, Version 3.4). Size of the CNV, total lesion and lesion components were classified as small $(\leqslant 4$ disc area $(\mathrm{DA}))$, medium $(>4$ to $<6 \mathrm{DA})$, and large ( $>6 \mathrm{DA}$ ). One DA was calculated as $2.54 \mathrm{~mm}^{2}$. (Greatest linear diameter of a lesion component with one DA in 
size corresponds to $1800 \mu \mathrm{m}$, if the lesion is round in shape.) The proportion of the fibrosis and haemorrhage to the total lesion was also determined on the colour fundus photographs.

Some eyes in predominantly haemorrhage group had considerable resolution of the haemorrhage 6 weeks after the treatment. However, only eyes with suspicious RPE tear or with consideration of therapy change underwent fluorescein angiography. Therefore, further information for this subgroup after resolution of the haemorrhage, based on the fluorescein angiography is limited.

The primary outcome was the occurrence of the RPE tear within 6 weeks ( \pm 2 weeks) following the treatment. The secondary goal was to identify potentially contributing factors, such as fluorescein angiographic subtype of the CNV lesion, morphology and size of the lesion components, fellow eye, and previous treatment that might have had an influence for the occurrence of the RPE tears.

Multiple linear regression modelling was conducted to investigate the effect of potentially predictive factors on the rate of RPE tears. Apart from the RPE tear, predictor variables in the model included baseline lesion size, baseline CNV size, lesion composition (haemorrhage, fibrosis), pretreatment and demographic characteristics of patients.

Insignificant terms for the risk of an RPE tear (predefined as $P>0.05$, effect Wald test) were eliminated from the model one at a time through a stepwise backward elimination process, when the main effect of any variable was not considered for elimination unless the interaction term involving the main-effect variable had already been eliminated. Model-adjusted probability was compared between the treatment groups for baseline categories (lesion seize and lesion type) using the same linear regression model.

To prove whether independent variables had a statistically significant effect on the risk of an RPE tear, the likelihood ratio test was used. The data set was analysed using the statistical software package JMP5.0.1.2 (SAS Institute Inc., Cary, NC, USA). The significance level was set to $\alpha=0.05$.

\section{Results}

Seven hundred and forty-six consecutive patients with neovascular AMD (768 eyes) were screened for this retrospective study. A total of 349 patients (359 eyes) were excluded from the study. One hundred seven of the excluded patients (117 eyes) had RAP or PCV. Fifty-five patients (55 eyes) had a failure from previous treatments and therefore switched from the previous treatment to the bevacizumab treatment. This group of patients has not been included into the analysis because of the absence of a baseline fluorescein angiography shortly before the bevacizumab injection. Detailed list of the excluded patients is given in the flow chart of the study (Figure 1, see Supplementary information).

The remaining 397 consecutive patients (409 eyes) met the eligibility criteria. Of these, 12 patients had bilateral bevacizumab treatment. Each treated eye was analysed separately. Baseline demographic characteristics of the patients are presented in Table 2.

About two-thirds of the patients were female (237) and one-third were male (160). In the RPE tear group the majority of patients was female (12 out of 15). Mean age of the non-RPE-tear group was 76.9 (95\% confidence interval (CI): 76.0-77.4) and 76.7 (95\% CI: 73.3-80.5) years in the RPE tear group.

\section{CNV lesion subtype}

Occult without classic CNV was found in $49.3 \%$ (202/409) of the eyes. Predominantly classic CNV, minimal classic $\mathrm{CNV}$, and predominantly haemorrhagic lesions were observed in 19.3 (79/409), $16.6(68 / 409)$, and $14.6 \%(60 / 409)$ of the eyes, respectively. Baseline lesion characteristics of the eyes at baseline are summarized in Table 3.

A total of $3.6 \%(15 / 409)$ of all eyes developed RPE tears (odds ratio (OR): 26.3; 95\% CI: 2.2-6.0). They occurred more frequently in predominantly haemorrhagic and occult without classic $\mathrm{CNV}$ groups with $6.6(4 / 60)$ and $4.9 \%(10 / 202)$ of the eyes, respectively. In the study collective, the data of the two types of the occult without $\mathrm{CNV}$ were not given separately. Type 2 occult lesions (late leakage of undetermined source) were detected in 22 eyes and no eyes in this subgroup had an RPE tear. Only one eye with minimal classic CNV had an RPE tear. No RPE tear was observed in eyes with predominantly classic CNV.

\section{Total lesion size}

Most of the eyes with neovascular AMD had a total lesion size less than 4DA 64.3\% (263/409). Medium size total lesion was in 9.0\% (37/409) and large size in $26.6 \%$ $(109 / 409)$ of the eyes. RPE tears occurred in medium and large total lesion with $5.4(2 / 37)$ and $7.3 \%(8 / 109)$ of the eyes, respectively. In $1.9 \%(5 / 263)$ of the eyes with small total lesion developed RPE tears. Medium and large size ( $>4 \mathrm{DA}$ ) of the total lesion was a positive predictor for the development of an RPE tear.

\section{CNV size}

Considering the size of the CNV lesion, 9.8\% (7/71) of the eyes developed an RPE tear if the CNV size was $6 \mathrm{DA}$ 


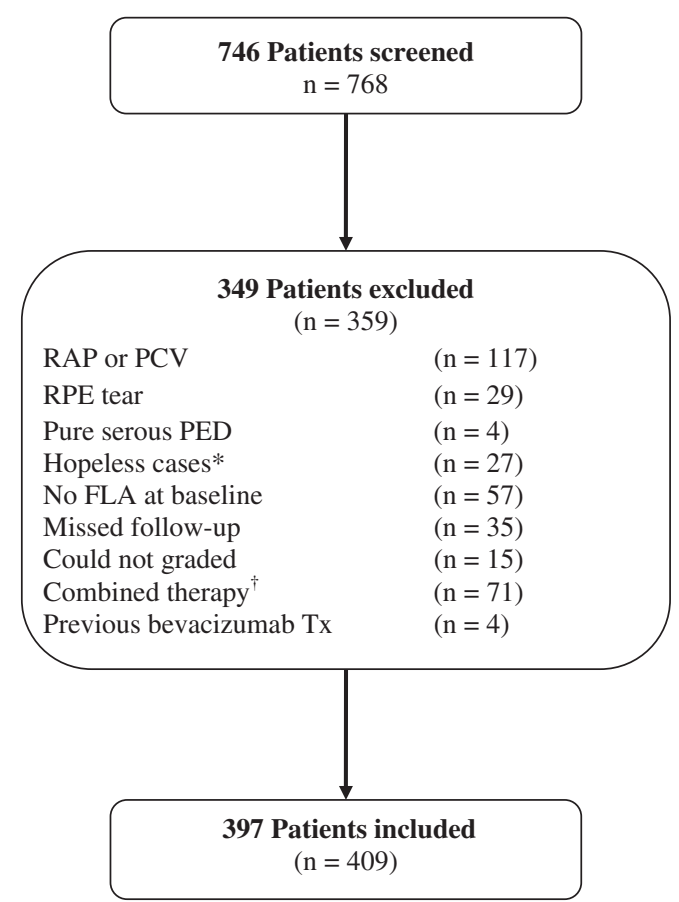

RAP = Retinal angiomatous proliferation PCV = Polypoidal choroidal vasculopathy RPE = Retinal pigment epithelium; PED = Pigment epithelial detachment; FLA = Fluorescein angiography; $\mathrm{Tx}=$ treatment.

* Defined as eyes with long standing large macular disciform lesions.

${ }^{\dagger}$ Defined as an additional therapy of the CNV or ocular surgery within 2 weeks of the intravitreal bevacizumab injection.

Figure 1 Flow chart of the study ( $n=$ eyes) (see the Supplementary information).

or larger. RPE tears occurred in $6.6 \%(2 / 30)$ of eyes with medium size of the CNV. Only $1.9 \%(6 / 308)$ of eyes with small CNV size developed RPE tear. The size of the CNV lesion influenced the occurrence of the RPE tear, however, without reaching statistical significance $(P=0.096)$.

\section{Subretinal haemorrhage and fibrosis}

If the subretinal haemorrhage was $50 \%$ or more of the total lesion, an RPE tear was detected in $6.9 \%$ (4/58) of the eyes. The larger proportion of the subretinal haemorrhage was statistically significant with RPE tears. The extent of the fibrosis was not found to be a significant factor for an RPE tear after intravitreal bevacizumab injection.

\section{Fellow eyes and previous treatment}

The proportion of the fellow eyes with CNV and/or CNV scar was $46.7 \%$. This was similar in the RPE tear and nonRPE tear group. In the RPE tear group 46.7\% (7/15) of the fellow eyes had CNV and/or CNV scar and 53.3\% (8/15) dry AMD. Previous treatment had $13.4 \%(2 / 15)$ of the eyes in the RPE tear group whereas $25.9 \%$ (102/409) of the eyes in the non-RPE tear group underwent previous therapies. The status of the fellow eyes and previous treatment did not show a significant difference between the RPE tear and non-RPE tear groups.

\section{Multiple linear regression modelling}

After considering all baseline parameters assessed to be independent risk factors, a statistical model was established by stepwise elimination of nonsignificant elements. This iteration resulted in a model, which just included CNV lesion type and total lesion size. The whole model test proved statistically significant $(P=0.003)$.

Separating minimal classic and predominantly classic CNV from occult without classic and predominant haemorrhagic $\mathrm{CNV}$ resulted in increased OR of 6.7 $(P=0.018)$ in favour of the latter group. When adapting to individual probability, we calculated an increase in risk of RPE tears of over $5 \%$ for occult or haemorrhagic $\mathrm{CNV}$ in comparison with a risk below $1 \%$ in minimal or 
Table 2 Demographic characteristics of patients at baseline

\begin{tabular}{|c|c|c|c|c|c|c|}
\hline & \multicolumn{2}{|c|}{$\begin{array}{l}\text { Non- } R P E \text { tear group } \\
(\mathrm{n}=394 \text { eyes }) \\
\text { (382 patients })\end{array}$} & \multicolumn{2}{|c|}{$\begin{array}{l}\text { RPE tear group } \\
\text { ( } \mathrm{n}=15 \text { eyes }) \\
(15 \text { patients })\end{array}$} & \multicolumn{2}{|c|}{$\begin{array}{l}\text { Total }(\mathrm{n}=409 \text { eyes }) \\
\quad(397 \text { patients })\end{array}$} \\
\hline & $\%(95 \% C I)$ & $\mathrm{n}$ & $\%(95 \% C I)$ & $\mathrm{n}$ & $\%(95 \% C I)$ & $\mathrm{n}$ \\
\hline Gender & & & & & $P=0.263$ & \\
\hline Women & $58.9(53.9-63.7)$ & 225 & $80.0(54.8-93.0)$ & 12 & $59.7(54.8-64.4)$ & 237 \\
\hline Men & $41.1(36.3-46.1)$ & 157 & $20.0(7.0-45.2)$ & 3 & $40.3(35.6-45.2)$ & 160 \\
\hline Age (years) & & & & & $P=0.924$ & \\
\hline Mean & 76.7 & & 76.9 & & 76.7 & \\
\hline Range & $56-95$ & & $67-86$ & & $56-95$ & \\
\hline $95 \% \mathrm{CI}$ & $76.0-77.4$ & & $73.3-80.5$ & & $76.0-77.4$ & \\
\hline Fellow eye & & & & & $P=0.800$ & \\
\hline Dry & $53.3(48.4-58.2)$ & 210 & $53.3(30.1-75.2)$ & 8 & $53.3(48.5-58.1)$ & 218 \\
\hline CNV & $17.0(13.6-21.0)$ & 67 & $20.0(7.0-45.1)$ & 3 & $17.1(13.8-21.1)$ & 70 \\
\hline CNV scar & $29.7(25.4-34.4)$ & 117 & $26.7(10.9-52.0)$ & 4 & $29.6(25.4-34.2)$ & 121 \\
\hline Previous treatment & & & & & $P=0.109$ & \\
\hline None & $74.1(69.6-78.2)$ & 292 & $86.7(62.1-96.3)$ & 13 & $74.5(70.1-78.6)$ & 305 \\
\hline Laser coagulaton & $2.5(1.4-4.6)$ & 10 & - & - & $2.4(1.3-4.4)$ & 10 \\
\hline PDT & $9.9(7.3-13.2)$ & 39 & - & - & $9.5(7.1-12.8)$ & 39 \\
\hline PDT + IVT or anti-VEGF & $5.8(3.9-8.6)$ & 23 & - & - & $5.6(3.8-8.3)$ & 23 \\
\hline IVT or anti-VEGF & $2.5(1.4-4.6)$ & 10 & - & - & $2.4(1.3-4.4)$ & 10 \\
\hline $\mathrm{rTPA}+\mathrm{Gas}$ & $3.6(2.1-5.9)$ & 14 & $6.7(1.2-29.8)$ & 1 & $3.7(2.2-6.0)$ & 15 \\
\hline Others $^{\mathrm{a}}$ & $1.5(0.6-3.3)$ & 6 & $6.7(1.2-29.8)$ & 1 & $1.7(0.8-3.5)$ & 7 \\
\hline
\end{tabular}

Abbreviations: $\mathrm{CI}=$ confidence interval; $\mathrm{CNV}=$ choroidal neovascularization; $\mathrm{FMT}=$ full macular translocation; IVT=intravitreal triamcinolone; $\mathrm{PDT}=$ photodynamic therapy; RPE $=$ retinal pigment epithelial; $\mathrm{rTPA}=$ recombinant tissue plasminogen factor; VEGF $=$ vascular endothelial growth factor.

$P$-value reflects the effect of Wald test.

a Other therapies includes transpupillary thermotherapy (three eyes), full macular translocation and later PDT (three eyes), PDT and IVT and later rTPA (one eye).

predominantly classic lesions. If assuming the lesion size as selecting criterion, a larger lesion resulted in significant smaller odds $(P=0.037)$. While the mean probability of RPE tears was $1.9 \%$ in small lesions, a lesion area over $4 \mathrm{DA}$ was associated with a likelihood estimate of approximately $7 \%$. Tables 4 and 5 show the data of the linear regression modelling and the mean probability of RPE tears given by the contributing factors of the model.

Clinical characteristics of the eyes with RPE tears are shown in Supplementary Table 6.

\section{Discussion}

This study shows that RPE tears may occur in $3.6 \%$ of eyes with neovascular AMD after single intravitreal bevacizumab injection within short-term follow-up. The occult without classic and the predominantly haemorrhagic subtype of CNV lesions and mediumto-large total lesion size were predictors for the development of RPE tears using multiple linear regression modelling.

The RPE tear is a well-known complication of neovascular AMD. It can be seen in the natural course of neovascular AMD and also after treatment, such as laser photocoagulation, photodynamic therapy, and various anti-VEGF therapies. ${ }^{29-34}$ Case series recently highlighted RPE tear increase after intravitreal bevacizumab treatment of neovascular AMD. ${ }^{14-26}$ Chan et al ${ }^{24}$ reported in a multicenter collective study 2.2\% RPE tears in 1064 eyes. In a subgroup of 123 eyes with PED, 21 eyes (17.1\%) had an RPE tear after intravitreal bevacizumab therapy. If only single injections are considered the incidence of RPE tears was $13 \%$ of the eyes with PED. Ronan et al ${ }^{23}$ reported from four centres a $0.08 \%$ RPE tear rate in a total of 1425 intravitreal injections. All cases with RPE tears had accompanying serous PED. The number of all treated eyes and a subgroup analysis of the CNV lesion were not given. They concluded that the estimated rate of the RPE tear could be underestimated, since not every chart of the study collective was specifically reviewed. 
Table 3 Baseline lesion characteristics of the study eyes

\begin{tabular}{|c|c|c|c|c|c|c|}
\hline & \multicolumn{2}{|c|}{ Non-RPE tear group $(\mathrm{n}=394$ eyes $)$} & \multicolumn{2}{|c|}{ RPE tear group $(\mathrm{n}=15$ eyes $)$} & \multicolumn{2}{|c|}{ Total $(\mathrm{n}=409$ eyes $)$} \\
\hline & $\%(95 \% C I)$ & $\mathrm{n}$ & $\%(95 \% C I)$ & $\mathrm{n}$ & $\%(95 \% C I)$ & $\mathrm{n}$ \\
\hline CNV lesion type & & & & & $P=0.016$ & \\
\hline Occult CNV & $48.7(43.8-53.7)$ & 192 & $66.7(41.7-84.8)$ & 10 & $49.4(44.6-54.2)$ & 202 \\
\hline Predominantly classic & $20.1(16.4-24.3)$ & 79 & - & - & $19.3(15.8-23.4)$ & 79 \\
\hline Minimal classic & $17.0(13.6-21.0)$ & 67 & $6.7(1.2-29.8)$ & 1 & $16.6(13.3-20.5)$ & 68 \\
\hline Predominantly haemorrhagic & $14.2(11.1-18.0)$ & 56 & $26.7(10.9-52.0)$ & 4 & $14.7(11.6-18.4)$ & 60 \\
\hline Total lesion size & & & & & $P=0.033$ & \\
\hline$\leqslant 4 \mathrm{DA}$ & $65.5(60.7-70.0)$ & 258 & $33.3(15.2-58.3)$ & 5 & $64.3(59.5-68.8)$ & 263 \\
\hline $4<$ and $<6 \mathrm{DA}$ & $8.9(6.5-12.1)$ & 35 & $13.3(15.2-58.3)$ & 2 & $9.0(6.6-12.2)$ & 37 \\
\hline$\geqslant 6 \mathrm{DA}$ & $25.6(21.6-30.2)$ & 101 & $53.3(30.1-75.2)$ & 8 & $26.7(22.6-31.1)$ & 109 \\
\hline CNV lesion size & & & & & $P=0.096$ & \\
\hline$\leqslant 4 \mathrm{DA}$ & $75.9(71.4-79.8)$ & 299 & $60.0(35.7-80.2)$ & 9 & $75.3(70.9-79.2)$ & 308 \\
\hline $4<$ and $<6$ DA & $7.4(5.2-10.4)$ & 29 & $6.7(1.2-29.8)$ & 1 & $7.3(5.1-10.3)$ & 30 \\
\hline$\geqslant 6 \mathrm{DA}$ & $16.8(13.4-20.8)$ & 66 & $33.3(15.2-58.3)$ & 5 & $17.4(14.0-21.3)$ & 71 \\
\hline Fibrosis/CNV & & & & & $P=0.766$ & \\
\hline$<25 \%$ & $91.1(87.9-93.5)$ & 359 & $93.3(70.2-98.8)$ & 14 & $91.2(88.0-93.6)$ & 373 \\
\hline$>25$ and $<50 \%$ & $1.8(0.9-3.6)$ & 7 & & & $1.7(0.8-3.5)$ & 7 \\
\hline$\geqslant 50 \%$ & $7.2(5.0-10.1)$ & 28 & $6.7(1.2-29.8)$ & 1 & $7.1(5.0-10.0)$ & 29 \\
\hline Haemorrhage/total lesion & & & & & $P=0.157$ & \\
\hline$<25 \%$ & $75.9(71.4-79.8)$ & 299 & $66.7(41.7-84.8)$ & 10 & $75.6(22.6-31.1)$ & 309 \\
\hline $25<$ and $\leqslant 50 \%$ & $10.4(7.8-13.8)$ & 41 & $6.7(1.2-29.8)$ & 1 & $10.3(7.7-13.6)$ & 42 \\
\hline$>50 \%$ & $13.7(10.7-17.5)$ & 54 & $26.7(10.9-52.0)$ & 4 & $14.2(11.1-17.9)$ & 58 \\
\hline
\end{tabular}

Abbreviations: $\mathrm{CI}=$ confidence interval; $\mathrm{CNV}=$ choroidal neovascularization; $\mathrm{DA}=$ disc area $\left(1 \mathrm{DA}=2.54 \mathrm{~mm}^{2}\right)$; $\mathrm{RPE}=$ retinal pigment epithelial.

Table 4 Linear regression model

\begin{tabular}{lcccc}
\hline Term & Estimate & $S E$ & $P^{\mathrm{a}}$ & $\begin{array}{c}\text { OR non-RPE } \\
\text { tear/RPE tear }\end{array}$ \\
\hline Intercept & 3.88 & 0.2 & $<0.0001$ & \\
$\begin{array}{l}\text { Lesion type } \\
\text { Minimal classic/predominantly } \\
\text { classic vs Occult-CNV/predominantly } \\
\text { haemorrhagic }\end{array}$ & 0.95 & 0.52 & 0.018 & 6.73 \\
$\begin{array}{l}\text { Lesion size } \\
\text { Small vs medium/large ( } \leqslant 4 \text { vs }>4 \mathrm{DA})\end{array}$ & 0.57 & 0.28 & 0.038 & 3.10 \\
\hline
\end{tabular}

Abbreviations: $\mathrm{CI}=$ confidence interval; $\mathrm{CNV}=$ choroidal neovascularization; $\mathrm{DA}=$ disc area; $\mathrm{OR}=$ odds ratio; $\mathrm{RPE}=$ retinal pigment epithelial.

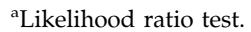

After bevacizumab therapy, Spandau and Jonas ${ }^{19}$ observed 6\% RPE tears in 63 eyes with PED secondary to AMD within 2 months. In our study collective of 409 eyes, RPE tear incidence was 3.6\%. Multiple linear regression modelling showed an increased risk of RPE tears for occult without classic and predominant haemorrhagic CNV (OR of 6.7). The risk of an RPE tear was over $5 \%$ for these subgroups. For minimal classic and predominantly classic CNV lesions, the risk was below $1 \%$. If the total lesion size was evaluated, the mean probability of RPE tears was $1.9 \%$ in small lesions ( $\leqslant 4 \mathrm{DA})$. In contrast, total lesion area over $4 \mathrm{DA}$ was associated with a likelihood estimate of approximately $7 \%$. The result of our and previous studies is in accordance with the high risk for the occult CNV subtype. Similar to the study of Chan et $a l^{24}$ we did not observe a significant association between the occurrence of RPE tears and the status of the fellow eye and previous 
Table 5 Probability of linear regression model

\begin{tabular}{|c|c|c|c|}
\hline & $\begin{array}{l}\text { No of } \\
\text { eyes }\end{array}$ & $\begin{array}{c}\text { Mean probability of } \\
\text { RPE tear }(\%)\end{array}$ & $\begin{array}{c}\text { Probability } \\
95 \% \text { CI }\end{array}$ \\
\hline \multicolumn{4}{|l|}{ Lesion type } \\
\hline Minimal classic & 68 & 0.74 & $0.20-1.28$ \\
\hline $\begin{array}{l}\text { Predominantly } \\
\text { classic }\end{array}$ & 79 & 0.62 & $0.13-1.13$ \\
\hline Occult-CNV & 202 & 5.18 & $4.87-5.49$ \\
\hline $\begin{array}{l}\text { Predominantly } \\
\text { haemorrhagic }\end{array}$ & 60 & 5.89 & $5.32-6.47$ \\
\hline \multicolumn{4}{|l|}{ Lesion size } \\
\hline$\leqslant 4 \mathrm{DA}$ & 263 & 1.90 & $1.64-2.15$ \\
\hline$>4$ and $<6 \mathrm{DA}$ & 37 & 7.06 & $6.38-7.75$ \\
\hline$\geqslant 6 \mathrm{DA}$ & 109 & 6.77 & $6.37-7.17$ \\
\hline
\end{tabular}

Abbreviations: $\mathrm{CNV}=$ choroidal neovascularization; $\mathrm{DA}=$ disc area; $\mathrm{RPE}=$ retinal pigment epithelial.

treatment within a short follow-up period. As reported in other studies, the vision in eyes with RPE tear did not show an acute decrease. ${ }^{23}$ In our study collective, median BCVA in those eyes was 20/200 before intravitreal bevacizumab injection and 20/60 at 6 weeks control. However, as RPE support for the function of the fovea is essential, long-term follow-up of these eyes is necessary to make a conclusive statement for the outcome. It should be mentioned that considerable variation of all these study designs makes it impossible to make a precise comparison of the data.

An important question is whether the RPE tears seen after bevacizumab administration are therapy-related or are the natural course of the disease. Limited data in the literature are available for the natural course of neovascular AMD with respect to the RPE tears. Casswell et $\mathrm{al}^{35}$ noted RPE tears in $10 \%$ of the eyes with RPE detachment over 5 years of follow-up. Similarly, Pauleikhoff et $a l^{36}$ reported RPE tears in $12.5 \%$ of eyes with vascular PED in a mean follow-up of 10.7 months. Chuang and Bird observed that within a follow-up period of up to 10 years, RPE tears in $35 \%$ of the eyes showed initial good visual acuity if the fellow eye presented with an RPE tear. ${ }^{37}$ The observed RPE tear rate in our study was $3.9 \%$. The occurrence of RPE tears was $4.9 \%$ in eyes with occult CNV and $6.6 \%$ in the predominantly haemorrhagic group. Since our study aimed to explore therapy-related RPE tears, follow-up time was limited to 6 weeks. Even though a direct comparison of our data with natural course studies is difficult, the detected frequency of RPE tears in some subgroups of our study seems to be high, especially considering the short-term follow-up period of 6 weeks.

In this study a strong association between the submacular haemorrhage and RPE tears was found. Few data are available assessing the relationship of the submacular haemorrhage with RPE tears in neovascular AMD. Reynders et $a l^{38}$ found scarred RPE tears in two of the 30 surgically extracted CNV in haemorrhagic AMD. Scupola et $a l^{39}$ reported in a retrospective study that 9 of 56 eyes developed RPE tears in the natural course of submacular haemorrhage secondary to AMD within a mean follow-up of 24 months. In addition, four eyes presented with an RPE tear at initial examination. An important question arose in all these studies, whether submacular haemorrhages might have obscured the RPE tears at the first presentation or even caused the haemorrhage. In our cases, the area of the RPE tears was not covered by submacular haemorrhages at baseline examination. Furthermore, all eyes in the predominantly haemorrhagic group had OCT examination before the treatment. Special attention was given in the OCT examination to whether an increased choroidal signal could be detected, which would suggest an RPE tear. And finally, since our study investigated RPE tears within 6 weeks after therapy, a reliable comparison with other reports is difficult.

The pathogenesis of RPE tears after intravitreal VEGF administration is not exactly known. The most accepted reason is the sharing force of the CNV on the RPE. ${ }^{22,30}$ Whether rapid resorption of the subretinal fluid after intravitreal bevacizumab injection is an additional factor for the sharing forces on the RPE, is still unanswered.

This study has some limitations. First it was retrospective. Second, the study collective included cases only from one centre. In contrast, possible selection bias of the cases could be avoided by doing so. Third, various lesion characteristics and previous treatment may have influenced the power of statistical analysis. Bearing these limitations in mind, in contrast, the study included cases seen in daily praxis. There is information about CNV lesions treated with intravitreal bevacizumab, which fall outside the indications for approved drugs, such as failed previous therapy, large or predominantly haemorrhagic lesions. Fourth, the outcome of multiple injections in the long-term follow-up was not addressed in this study. Therefore, no information is given to establish the cumulative risk profile of RPE tears after multiple treatments. Fifth, not every patient at 6 weeks had a fluorescein angiography that may have led to an underestimation of the incidence of the RPE tear. In contrast, missed cases of RPE tear should be minimal, as every eye had fundus photography, OCT imaging of the macula in addition to the slit lamp ophthalmoscopy with dilated pupil. Sixth, the determination of the status of the fellow eye may have limitations, since a scar formation of previous RPE tears might likely exclude a precise recognition of this feature. Finally, cases of RAP and PCV were excluded since they may have another clinical course than neovascular AMD. 
This study addresses the occurrence of RPE tears with a short time interval after single intravitreal bevacizumab injection. Repeated intravitreal injections are often needed, so the number of related complications may increase in long-term follow-up after multiple treatments. Our study reveals that eyes with some characteristics of CNV lesions have higher risks for the occurrence of RPE tears after the treatment. Identifying these factors preoperatively is of utmost importance to clarify the risk profile of the candidates for intravitreal bevacizumab treatment.

\section{References}

1 Klein R, Klein BEK, Linton KLP. Prevalence of age-related maculopathy. The beaver dam eye study. Ophthalmology 1992; 99: 933-943.

2 Kvanta A, Algvere PV, Berglin L, Seregard S. Subfoveal fibrovascular membranes in age-related macular degeneration expresses vascular endothelial growth factor. Invest Ophthalmol Vis Sci 1996; 37: 1929-1934.

3 Kliffen M, Sharma HS, Mooy CM, Kerkvliet S, de Jong PT. Increased expression of angiogenic growth factors in agerelated maculopathy. Br J Ophthalmol 1997; 81: 154-162.

4 Krzystolik MG, Afshari MA, Adamis AP, Gaudreault J, Gragoudas ES, Michaud NA et al. Prevention of experimental choroidal neovascularization with intravitreal antivascular endothelial growth factor antibody fragment. Arch Ophthalmol 2002; 120: 338-346.

5 Gragoudas ES, Adamis AP, Cunningham Jr ET, Feinsod M, Guyer DR. VEGF Inhibition Study in Ocular Neovascularization Clinical Trial Group. Pegaptanib for neovascular age-related macular degeneration. $N$ Engl J Med 2004; 351: 2805-2816.

6 Brown DM, Kaiser PK, Michels M, Soubrane G, Heier JS, Kim RY et al. Ranibizumab versus verteporfin for neovascular age-related macular degeneration. $N$ Engl J Med 2006; 355: 1432-1444.

7 Rosenfeld PJ, Brown DM, Heier JS, Boyer DS, Kaiser PK, Chung CY et al. Ranibizumab for neovascular age-related macular degeneration. N Engl J Med 2006; 355: 1419-1431.

8 Rosenfeld PJ, Moshfeghi AA, Puliafito CA. Optical coherence tomography findings after an intravitreal injection of bevacizumab (avastin) for neovascular agerelated macular degeneration. Ophthalmic Surg Lasers Imaging 2005; 36: 331-335.

9 Avery RL, Pieramici DJ, Rabena MD, Castellarin AA, Nasir MA, Giust MJ. Intravitreal bevacizumab (Avastin) for neovascular age-related macular degeneration. Ophthalmology 2006; 113: 363-372.

10 Bashshur ZF, Bazarbachi A, Schakal A, Haddad ZA, El Haibi CP, Noureddin BN. Intravitreal bevacizumab for the management of choroidal neovascularization in age-related macular degeneration. Am J Ophthalmol 2006; 142: 1-9.

11 Spaide RF, Laud K, Fine HF, Klancnik Jr JM, Meyerle CB, Yannuzzi LA et al. Intravitreal bevacizumab treatment of choroidal neovascularization secondary to age-related macular degeneration. Retina 2006; 26: 383-390.

12 Rich RM, Rosenfeld PJ, Puliafito CA, Dubovy SR, Davis JL, Flynn Jr HW et al. Short-term safety and efficacy of intravitreal bevacizumab (Avastin) for neovascular agerelated macular degeneration. Retina 2006; 26: 495-511.

13 Luke M, Warga M, Ziemssen F, Gelisken F, Grisanti S, Schneider Tet al. Effects of bevacizumab on retinal function in isolated vertebrate retina. $\mathrm{Br} J$ Ophthalmol 2006; 90: 1178-1182, . e-pub 5 June 2006.

14 Maturi RK, Bleau LA, Wilson DL. Electrophysiologic findings after intravitreal bevacizumab (Avastin) treatment. Retina 2006; 26: 270-274.

15 Nicolo M, Ghiglione D, Calabria G. Retinal pigment epithelial tear following intravitreal injection of bevacizumab (Avastin). Eur J Ophthalmol 2006; 16: 770-773.

16 Meyer CM, Mennel S, Schmidt JS, Kroll P. Acute retinal pigment epithelial tear following intravitreal bevacizumab (Avastin) injection for occult choroidal neovascularisation secondary to age related macular degeneration. $\mathrm{Br} \mathrm{J}$ Ophthalmol 2006; 90: 1207-1208.

17 Bakri SJ, Patel SP. Retinal pigment epithelial tear following intravitreal bevacizumab. Eye 2007; 21: 424-425.

18 Gelisken F, Ziemssen F, Voelker M, Bartz-Schmidt KU. Retinal pigment epithelial tear following intravitreal bevacizumab injection for neovascular age-related macular degeneration. Acta Ophthalmol Scand 2006; 84: 833-834.

19 Spandau UH, Jonas JB. Retinal pigment epithelium tear after intravitreal bevacizumab for exudative age-related macular degeneration. Am J Ophthalmol 2006; 142: 1068-1070.

20 Shah CP, Hsu J, Garg SJ, Fischer DH, Kaiser R. Retinal pigment epithelial tear after intravitreal bevacizumab injection. Am J Ophthalmol 2006; 142: 1070-1072.

21 Gamulescu MA, Framme C, Sachs H. RPE-rip after intravitreal bevacizumab (Avastin) treatment for vascularised PED secondary to AMD. Graefes Arch Clin Exp Ophthalmol 2007; 245: 1037-1040, e-pub 21 February 2007.

22 Chang LK, Sarraf D. Tears of the retinal pigment epithelium. An old problem in a new Era. Retina 2007; 27: 523-534.

23 Ronan SM, Yoganathan P, Chien FY, Corcostegui IA, Blumenkranz MS, Deramo VA et al. Retinal pigment epithelium tears after intravitreal injection of bevacizumab (avastin) for neovascular age-related macular degeneration. Retina 2007; 27: 535-540.

24 Chan CK, Meyer CH, Gross JG, Abraham P, Nuthi AS, Kokame GT et al. Retinal pigment epithelial tears after intravitreal bevacizumab injection for neovascular agerelated macular degeneration. Retina 2007; 27: 541-551.

25 Shabeeba R, Hannan SR, Madhusudhana KC, Lotery AJ Newsom RSB. Retinal pigment epithelial tear following bevacizumab for choroidal neovascular membrane due to age-related macular degeneration. Br J Ophthalmol 2007; 91: 977-978.

26 Mathews JP, Jalil A, Lavin MJ, Stanga PE. Retinal pigment epithelial tear following intravitreal injection of bevacizumab (avastin): optical coherence tomography and fluorescein angiographic findings. Eye 2007; 21: 1004-1005.

27 Macular Photocoagulation Study Group. Subfoveal neovascular lesions in age-related macular degeneration: guidelines for evaluation and treatment in the Macular Photocoagulation Study. Arch Ophthalmol 1991; 109: 1242-1257.

28 Verteporfin Roundtable Participants. Guidelines for using verteporfin (Visudyne) in photodynamic therapy for choroidal neovascularization due to age-related macular degeneration and other causes: update. Retina 2005; 25: 119-134. 
29 Hoskin A, Bird AC, Sehmi K. Tears of detached retinal pigment epithelium. Br J Ophthalmol 1981; 65: 859-865.

30 Gass JD. Retinal pigment epithelial rip during krypton red laser photocoagulation. Am J Ophthalmol 1984; 98: 700-706.

31 Gelisken F, Inhoffen W, Partsch M, Schneider U, Kreissig I. Retinal pigment epithelial tear after photodynamic therapy for choroidal neovascularization. Am J Ophthalmol 2001; 131: 518-520.

32 Goldstein M, Heilweil G, Barak A, Loewenstein A. Retinal pigment epithelial tear following photodynamic therapy for choroidal neovascularization secondary to AMD. Eye 2005; 19: 1315-1324.

33 Dhalla MS, Blinder KJ, Tewari A, Hariprasad SM, Apte RS Retinal pigment epithelial tear following intravitreal pegaptanib sodium. Am J Ophthalmol 2006; 141: 752-754.

34 Amselem L, Pulido JS, Diaz-Llopis M, Cervera E, Montero J. Retinal pigment epithelial tear following ranibizumab use. Eye 2007; 21(6): 846-847, e-pub ahead of print 26 January.
35 Casswell AG, Kohen D, Bird AC. Retinal pigment epithelial detachments in the elderly: classification and outcome. Br J Ophthalmol 1985; 69: 397-403.

36 Pauleikhoff D, Loeffert D, Spital G, Radermacher M, Dohrmann J, Lommatzsch A et al. Pigment epithelial detachment in elderly. Clinical differentiation, natural course and pathogenetic implications. Graefes Arch Clin Exp Ophthalmol 2002; 533-538.

37 Chuang EL, Bird AC. Bilaterality of tears of the retinal pigment epithelium. Br J Ophthalmol 1988; 72: 918-920.

38 Reynders S, Lafaut BA, Aisenbrey S, Broecke CV, Lucke K, Walter $\mathrm{P}$ et al. Clinicopathologic correlation in hemorrhagic age-related macular degeneration. Graefes Arch Clin Exp Ophthalmol 2002; 240: 279-285.

39 Scupola A, Coscas G, Soubrane G, Balestrazzi E. Natural history of macular subretinal hemorrhage in age-related macular degeneration. Ophthalmologica 1999; 213: $97-102$

Supplementary Information accompanies the paper on Eye website (http://www.nature.com/eye) 